\title{
Paradas durante la caminata de 6 minutos y su correlación con nuevas mediciones de la prueba en pacientes con enfermedad pulmonar obstructiva crónica
}

\author{
Stops during the six-minute walk test and their \\ correlation with new measurements of the test in \\ patients with obstructive pulmonary disease
}

\author{
María José Fernández-SÁnCheZ ${ }^{(1,2)}$; Milena García(2); XIMENA CorRea-Ríos ${ }^{(2)}$; \\ ALEJANDRA CAÑAS(2); JAVIER IVÁN LASSO ${ }^{(2)}$; JUAN RICARDO LUTZ(2); CLAUDIO VILLAQUIRÁN(2); \\ CARLos Celis ${ }^{(2)}$
}

\section{Resumen}

MARCO TEÓRICO: se ha reportado que la presencia de paradas involuntarias durante la prueba de caminata de 6 minutos se asocia con un incremento en la mortalidad en los pacientes con enfermedad pulmonar obstructiva crónica. Sin embargo, la relación de las paradas con otras determinaciones de la prueba de caminata de 6 minutos como velocidad, trabajo, el producto distancia-saturación y la desaturación inducida por el ejercicio, aún no se ha establecido.

OBJETIVOS: determinar la correlación de las paradas involuntarias con variables clínicas recolectadas de forma rutinaria en la PC6M, así como con las nuevas determinaciones derivadas de la prueba, en pacientes con enfermeda pulmonar obstructiva crónica.

MATERIALES Y MÉTODOS: se realizó un estudio observacional, analítico, retrospectivo, en el que se revisaron los reportes de pruebas de caminanta de 6 minutos de 129 sujetos con enfermedad pulmonar obstructiva crónica. Las determinaciones derivadas de la prueba fueron comparadas entre los pacientes que presentaron paradas y quienes no; se realizaron correlaciones entre ellas y se identificaron los predictores de paradas por medio de análisis de regresión logística.

RESULTADOS: 30 pacientes tuvieron paradas involuntarias durante la prueba; en este grupo se observó al final que los puntajes de disnea y fatiga, así como la frecuencia cardiaca, la frecuencia respiratoria y la tensión arterial sistólica final fueron significativamente mayores que en el grupo que no tuvo paradas, mientras que la saturación arterial de oxigeno fue significativamente menor $(p<0,001)$; las nuevas variables derivadas: distancia, velocidad, trabajo y el producto distancia-saturación fueron menores $(p<0,001)$ en los sujetos que pararon, encontrando una buena correlación con ellas. En el análisis de regresión, las variables que permanecieron significativas para la presencia de paradas fueron: saturación final, distancia recorrida, velocidad, trabajo, DDR y producto distancia-saturación.

CONCLUSIONES: en los pacientes con enfermedad pulmonar obstructiva crónica estable que realizan una prueba de caminata de 6 minutos, la presencia de paradas se asocia con menor distancia caminada, trabajo, DSP y saturación al final de la prueba. El cálculo de estas nuevas variables, así como el registro de paradas durante la PC6M podría refinar la capacidad pronóstica de la prueba en pacientes con enfermedad pulmonar obstructiva crónica.

Palabras clave: caminata de 6 minutos, enfermedad pulmonar obstructiva crónica, paradas involuntarias, distancia.

\footnotetext{
Abstract

THEORETICAL FRAME: it has been reported that involuntary stops during the six-minute walk test are associated with increased mortality in patients with chronic obstructive pulmonary disease. However, the relationship between the stops and other determinations of the six-minute walk test, such as speed, work, the distance-saturation product, and exercise-induced desaturation, has not yet been established.
}

${ }^{(1)}$ Departamento de Fisiología y Farmacología, Facultad de Medicina, Pontificia Universidad Javeriana, Bogotá, D.C., Colombia; Departamento de Medicina Interna, Hospital Universitario San Ignacio, Bogotá, D.C., Colombia.

${ }^{(2)}$ Unidad de Neumología, Hospital Universitario San Ignacio, Bogotá, D.C., Colombia.

Correspondencia: Carlos Celis, correo electrónico: cacelis.neumo@gmail.com Recibido: 16/05/15, Aceptado: 26/05/15 
OBJECTIVES: to determine the correlation between involuntary stops and clinical variables gathered routinely in the six-minute walk test, as well as the new determinations derived from the test, in patients with chronic obstructive pulmonary disease.

MATERIALS AND METHODS: an observational, analytic, retrospective study was carried out, in which the reports of six-minute walk tests from 129 subjects with chronic obstructive pulmonary disease were reviewed. The determinations derived from the test were compared between the patients who had stops and those who hadn't; correlations between them were made, and predictors of stops were identified by logistic regression anaylysis.

RESULTS: 30 patients had involuntary stops during the test; in this group, it was observed in the end that scores of dyspnea and fatigue, as well as heart rate, respiratory rate, and final systolic arterial blood pressure were significantly higher than those found in the group that did not have stops, while arterial blood oxygen saturation was significantly lower $(p<0,001)$; the new variables derived: distance, speed, work, and the distance-saturation produc were lower $(\mathrm{p}<0,001)$ in the subjects who stopped; a good correlation with them was found. On regression analysis, the variables that remained significant for the presence of stops were: final saturation, distance walked, speed, work, DDR, and distance-saturation product.

CONCLUSIONS: in patients with stable chronic obstructive pulmonary disease who undergo the six-minute walk test, stops are associated with less distance walked, work, DSP, and saturation at the end of the test. Calculation of these new variables, together with registration of stops during the six-minute walk test, might refine the prognostic capability of the test in patients with chronic obstructive pulmonary disease.

Keywords: six-minute walk test, chronic obstructive pulmonary disease, involuntary stops, distance.

\section{Introducción}

La enfermedad pulmonar obstructiva crónica (EPOC) es una de las principales causas de morbilidad en todo el mundo y se proyecta como la tercera causa de muerte y la quinta de discapacidad en años de vida ajustados en 2020 (1). En Colombia, su prevalencia en mayores de 40 años es de $8,9 \%$ (IC95\% 8,2-9,7\%), según los resultados del estudio multicéntrico PREPOCOL, siendo el antecedente de tabaquismo y la exposición por más de 10 años al humo de leña, los principales factores de riesgo (2).

La recientemente publicada "Guía de Práctica Clínica Basada en la Evidencia para la Prevención, Diagnóstico, Tratamiento y Seguimiento de la EPOC", dio un giro importante en la evaluación del paciente, al recomendar la escala multidimensional BODE como estrategia de clasificación inicial de gravedad, para la evaluación de la respuesta al tratamiento y en el seguimiento (3).

Dicho índice incluye el índice de masa corporal como reflejo del compromiso sistémico de la enfermedad, el grado espirométrico de obstrucción, la disnea como síntoma principal y la distancia caminada en la prueba de caminata de 6 minutos (PC6M) como método de evaluación de la capacidad de ejercicio (4), y es un predictor independiente de riesgo de exacerbaciones, mortalidad y necesidad de trasplante pulmonar (5-7).

La incapacidad de ejercicio en los pacientes con EPOC, que puede presentarse desde las fases iniciales de la enfermedad, es un acontecimiento definitivo en la historia natural de la entidad al tener influencia en la presencia de síntomas, el deterioro de la calidad de vida relacionada con la salud, la frecuencia de exacerbaciones severas (hospitalizaciones) y la supervivencia $(8,9)$.

De esta forma, el alto valor predictivo de la baja capacidad para el ejercicio es atribuible al origen multifactorial de ésta: a) limitación ventilatoria por disfunción mecánica; b) alteración del intercambio de gases; c) incremento inadecuado del gasto cardíaco durante el ejercicio, y d) disfunción muscular periférica (10). Este carácter multifactorial, junto con las limitaciones de la función pulmonar medida en condiciones de reposo, confirma la necesidad de incluir la evaluación de la capacidad de ejercicio dentro de los estudios realizados en los pacientes con EPOC (11).

La PC6M es un examen fácil de realizar, ha sido bien estandarizada (12-14) y la distancia recorrida en la prueba (metros) ha demostrado ser un predictor independiente de supervivencia que permite estimar el riesgo de hospitalizaciones (15). Así mismo, ha 
mostrado un alto valor pronóstico de la evolución postoperatoria en pacientes con EPOC candidatos a cirugía de reducción de volumen al identificar individuos con evolución desfavorable (16).

Además de la distancia caminada, se han reportado otras determinaciones derivadas de la PC6M, tales como la velocidad, el trabajo, el producto distancia-saturación (DSP), la desaturación de oxígeno inducida por el ejercicio y la cantidad de paradas involuntarias, las cuales también podrían tener un valor pronóstico en la identificación de pacientes con EPOC y riesgo de desenlaces adversos (17).

Recientemente, Andrianopoulos et al. reportaron un análisis de los datos de la cohorte ECLIPSE, llevado a cabo en 2010 en pacientes con EPOC moderada a severa (87\%), quienes fueron seguidos durante tres años con el fin de determinar la asociación de estos nuevos índices derivados de la PC6M y los riesgos de mortalidad y hospitalización a lo largo del seguimiento (17).

Los autores señalan que los sujetos que mostraron distancia <334 metros (HR 2,30; IC95\% 1,622,38; $<<0,001)$, velocidad $\leq 0,9 \mathrm{~m} / \mathrm{s}$ (HR 2,15; IC95\% 1,52-3,04; $<<0,001)$, trabajo $\leq 20.000 \mathrm{~m} / \mathrm{kg}$ (HR 2,17; IC95\% 1,51-3,12; $\mathrm{p}<0,001)$, producto distanciasaturación $\leq 290 \mathrm{~m} \%$ (HR 2,70; IC95\% 1,89-3,86; $\mathrm{p}<0,001$ ), saturación final $\leq 88 \%$ (HR 1,75; IC95\% $1,25-2,44 ; \mathrm{p}<0,001)$ o tuvieron paradas durante la prueba (HR 1,99; IC95\% 1,20-3,30; $<<0,001$ ), presentaron mayor riesgo de mortalidad (17).

Así mismo, aquellos con saturación final $\leq 88 \%$ (HR 1,25; IC95\% 1,04-1,51; p<0,001) o paradas (HR 1,70; IC95\% 1,20-2,39; $\mathrm{p}=0,003$ ) tuvieron un mayor riesgo de hospitalización (17).

El propósito de este estudio es evaluar la correlación de la presencia de paradas involuntarias durante la PC6M con la distancia y los otros cálculos derivados de la prueba, siendo nuestra hipótesis la presencia de una correlación importante entre ellas. Esta información permitirá acercarse a las bases fisiológicas que relacionan dichas determinaciones y servirá como punto de partida para su empleo combinado como una estrategia más refinada de pronóstico en el paciente con EPOC.

\section{Metodología}

\section{Diseño}

Se realizó un estudio clínico prospectivo observacional de tipo cohorte retrospectiva, en el que se encontró el vínculo entre variables demográficas y/o nuevas variables derivadas de la PC6M y presencia de paradas involuntarias durante la PC6M en pacientes con diagnóstico de EPOC remitidos para realización de la prueba en la Unidad de Neumología del Hospital Universitario San Ignacio en Bogotá, Colombia entre los años 2012 a 2015. Para su ejecución se sometió a aprobación el protocolo del estudio por el comité de investigaciones del Hospital Universitario San Ignacio y de la Pontificia Universidad Javeriana. Teniendo en cuenta que los cálculos de las nuevas variables derivadas de la PC6M se realizaron a partir de registros existentes, no se consideró necesario firmar un consentimiento informado adicional. El estudio fue aprobado por el Comité de Ética e Investigación del Hospital Universitario San Ignacio.

\section{Población}

El estudio se llevó a cabo en pacientes de la Unidad de Neumología del Hospital Universitario San Ignacio, en Bogotá, Colombia, quienes fueron manejados por el personal asistencial de la unidad, sin ninguna interferencia en su plan diagnóstico y terapéutico por el grupo de investigadores. Se revisaron los registros de pacientes con diagnóstico médico de EPOC, remitidos para realización de PC6M, sin antecedente de exacerbación severa (hospitalización) en las últimas seis semanas. Se excluyeron sujetos con condiciones que impidieran realizar de forma completa la PC6M, como enfermedad cardiovascular activa e hipertensión arterial no controlada, o aquellos con limitación para realizar la prueba por comorbilidades osteomusculares o vasculares periféricas asociadas.

\section{Protocolo}

Una vez revisada la información se seleccionaron los pacientes que cumplieron los criterios de inclusión. Dos investigadores extractaron en un formato prediseñado y de forma independiente las variables demográficas y las registradas en 
la PC6M y calcularon las nuevas determinaciones tales como: velocidad (metros/segundo), trabajo (metros/kg), DSP (metros\%), EID (desaturación inducida por ejercicio) (si o no) y paradas (si o no). Uno de los coinvestigadores se encargó de revisar la coherencia de la información registrada y los cálculos de cada paciente. Ante cualquier inconsistencia o evidencia de falta de datos, se retornó al caso específico a fin de efectuar la corrección de los mismos.

\section{Mediciones}

La PC6M se llevó cabo de acuerdo con las guías de las sociedades ATS/ERS (del inglés American Thoracic Society/European Respiratory Society) de 2002 (12) y el documento de Estandarización de Procedimientos Operativos de pruebas de ejercicio en enfermedad respiratoria crónica, publicado en $2014(13,14)$.

El paciente recibió información del evaluador responsable de las características de la prueba de caminata de 6 minutos; se verificó la lista de chequeo de indicaciones y contraindicaciones de la prueba y se diligenció el consentimiento informado, explicado y firmado por el paciente, su tutor legal o familiar más cercano como es usual antes de este examen y no se realizó la PC6M al paciente hasta tanto no se contara con dicha autorización.

Después de informar al paciente acerca de las características de la prueba, se tomaron signos vitales como frecuencia cardiaca (FC), frecuencia respiratoria (FR), tensión arterial sistólica (TAS), tensión arterial diastólica (TAD), saturación arterial de oxígeno $\left(\mathrm{SaO}_{2}\right)$, y se registró el grado de disnea y de fatiga de las extremidades inferiores según la escala Borg en condiciones basales. No se permitió hacer antes de la prueba. Las mediciones se realizaron con el paciente sentado durante 10 minutos antes de la prueba. Antes de comenzar la caminata se le recordó al paciente el objetivo de la prueba, y una vez situados en uno de los extremos del trayecto, se dio la señal verbal de empezar a caminar.

La prueba se llevó a cabo en un corredor recto de 30 metros de largo bajo la guía de una fisioterapeuta respiratoria, y se hizo una práctica con las personas que previamente no habían realizado el examen (13, 14). Durante el ejercicio, la fisioterapeuta informaba a los pacientes cuándo había transcurrido cada minuto y les animaba a continuar.

Se les permitió detenerse si aparecían síntomas como disnea, dolor torácico, mareo, sudoración o fatiga en miembros inferiores y estas paradas involuntarias fueron registradas; sin embargo, se les pidió reanudar la caminata lo más pronto posible, si podían.

En caso de parada y después de terminar la caminata, se determinaron mediciones de $\mathrm{SaO}_{2}$, TAS, TAD, FC, FR, disnea y fatiga. $\mathrm{La} \mathrm{SaO}_{2}$ y la FC se registraron a través de un oxímetro de pulso portátil (Pulsox-2, Konica Minolta, Japón); la tensión arterial se registró mediante tensiómetro manual calibrado, en tanto que la disnea y la fatiga fueron calificadas por el paciente empleando la escala de Borg (puntajes de 0 a 10). Al final de la prueba se registró la distancia recorrida (metros) y el tiempo (segundos) de duración de la caminata. La saturación de oxígeno y las frecuencias cardiaca y respiratoria también se registraron cada minuto a lo largo del examen $(13,14)$.

\section{Análisis estadístico}

Los sujetos fueron clasificados como "paradores" y "no paradores" de acuerdo con la presencia o no de paradas involuntarias durante la PC6M.

Los resultados se muestran como promedio $( \pm$ desviación estándar) o proporción (\%). La diferencia entre grupos se calculó mediante análisis de varianza de Kolmogorov-Smirnov, test t Student, test de Fisher y test de Chi-cuadrado de acuerdo como fuera apropiado (según variables categóricas y continuas y homogeneidad de varianza).

Se realizaron correlaciones de Pearson (datos paramétricos) y de Spearman (datos no paramétricos) para establecer la correlación entre las paradas en la caminata con los datos demográficos y las nuevas determinaciones de la PC6M.

Así mismo, se llevó a cabo un análisis de regresión logística binaria cuasibinomial para las variables independientes. La variable con mayor significancia 
del análisis se analizó con una curva ROC y se calculó su área bajo la curva (AUC). La hipótesis nula en todos los casos fue rechazada con un nivel de significancia de $p<0,05$. Los análisis estadísticos se hicieron empleando el programa SPSS ${ }^{\circledR}$ versión 18 (SPSS, Inc., Chicago, IL, EE.UU) y PAST ${ }^{\circledR}$ versión 3 (Universidad de Oslo, Noruega).

\section{Resultados}

\section{Sujetos}

El grupo de estudio incluyó un total de 129 pacientes remitidos con diagnóstico de EPOC, reclutados desde 2012 a 2015 y a quienes se les realizó una PC6M.
El 60\% $(n=78)$ de los participantes fueron hombres, con promedio de edad de 72,3 $\pm 8,9$ años (edad mínima de 40 años y máxima de 91 años). La talla promedio fue de $160,8 \pm 9,3 \mathrm{~cm}$, el peso promedio de $64,7 \pm 9,7 \mathrm{~kg}$ y el índice de masa corporal (IMC) de $25,1 \pm 4,1 \mathrm{~kg} / \mathrm{m}^{2}$ en promedio; el $55 \%(\mathrm{n}=71)$ de $\mathrm{los}$ pacientes estaba en el rango de normalidad (IMC 18$\left.25 \mathrm{~kg} / \mathrm{m}^{2}\right)$ y el $34 \%(\mathrm{n}=44)$ en sobrepeso (IMC 25,1 $30,0 \mathrm{~kg} / \mathrm{m}^{2}$ ) (tabla 1$)$.

\section{Resultados variables clínicas}

$\mathrm{Al}$ inicio de la prueba el promedio de la disnea por escala de Borg fue mínimo (0,4 $\pm 1,1$ puntos) y el de fatiga muy leve $(0,5 \pm 1,2$ puntos). Los promedios de saturación, FC, FR, TAS y TAD en la población general,

Tabla 1. Características demográficas y basales de los pacientes clasificados según presencia de paradas en la caminata de 6 minutos.

\begin{tabular}{|c|c|c|c|c|}
\hline Características & $\begin{array}{c}\text { Total } \\
(n=129)\end{array}$ & $\begin{array}{l}\text { Pararon } \\
(n=30)\end{array}$ & $\begin{array}{c}\text { No pararon } \\
(n=99)\end{array}$ & P valor \\
\hline Mujeres, n (\%) & $51(39,5)$ & $13(43,3)$ & $38(38,3)$ & 0,63 \\
\hline Edad, años & $72,3 \pm 8,9$ & $75,1 \pm 8,0$ & $71,4 \pm 9,0$ & 0,06 \\
\hline Talla, cm & $160,8 \pm 9,3$ & $161,1 \pm 10,8$ & $160,7 \pm 8,8$ & 0,83 \\
\hline Peso, kg & $64,7 \pm 9,7$ & $64,1 \pm 10,9$ & $64,8 \pm 9,4$ & 0,72 \\
\hline $\mathrm{IMC}, \mathrm{kg} / \mathrm{m}^{2}$ & $25,1 \pm 4,1$ & $24,8 \pm 4,4$ & $25,2 \pm 4,0$ & 0,72 \\
\hline Disnea inicial, Borg & $0,4 \pm 1,1$ & $0,91 \pm 1,7$ & $0,3 \pm 0,7$ & 0,06 \\
\hline Disnea final, Borg & $3,8 \pm 2,7$ & $6,3 \pm 2,9$ & $3,1 \pm 2,1$ & $<0,001$ \\
\hline Fatiga inicial, Borg & $0,5 \pm 1,2$ & $1,1 \pm 1,8$ & $0,3 \pm 0,9$ & $<0,05$ \\
\hline Fatiga final, Borg & $3,6 \pm 2,7$ & $6,4 \pm 2,8$ & $2,8 \pm 2,0$ & $<0.001$ \\
\hline $\mathrm{SaO}_{2}$ inicial, \% & $89 \pm 9$ & $86,6 \pm 5,5$ & $89,2 \pm 10$ & 0,06 \\
\hline $\mathrm{SaO}_{2}$ final, $\%$ & $81 \pm 9$ & $75,6 \pm 11$ & $82,7 \pm 7$ & $<0,01$ \\
\hline FC inicial, Ipm & $78 \pm 14$ & $81 \pm 15$ & $76 \pm 13$ & 0,1 \\
\hline FC final, Ipm & $107 \pm 17$ & $110 \pm 17$ & $107 \pm 16$ & $<0,01$ \\
\hline FR inicial, rpm & $17 \pm 2$ & $18 \pm 2$ & $16 \pm 2$ & $<0,01$ \\
\hline FR final, rpm & $38 \pm 4$ & $40 \pm 3$ & $37 \pm 4$ & $<0,001$ \\
\hline TAS inicial, $\mathrm{mm} \mathrm{Hg}$ & $121 \pm 16$ & $119 \pm 16$ & $121 \pm 16$ & 0,45 \\
\hline TAS Final, $\mathrm{mm} \mathrm{Hg}$ & $137 \pm 18$ & $145 \pm 21$ & $135 \pm 17$ & $<0,05$ \\
\hline TAD Inicial, mm Hg & $72 \pm 9$ & $73 \pm 9$ & $72 \pm 9$ & 0,87 \\
\hline TAD Final, mm Hg & $78 \pm 10$ & $80 \pm 11$ & $78 \pm 9$ & 0,26 \\
\hline
\end{tabular}


Tabla 2. Datos de la caminata de 6 minutos en pacientes categorizados según paradas.

\begin{tabular}{|lrrr|}
\hline \multicolumn{1}{|c}{ Características } & $\begin{array}{c}\text { Pararon } \\
(\mathbf{n}=\mathbf{2 3})\end{array}$ & $\begin{array}{c}\text { No pararon } \\
(\mathbf{n}=\mathbf{9 9 )}\end{array}$ & P valor \\
EID, $\mathrm{n}(\%)$ & $26(86,6)$ & $76(76,7)$ & 1,99 \\
Distancia, $\mathrm{m} \pm \mathrm{DE}$ & $252 \pm 90$ & $395 \pm 108$ & $<0,001$ \\
Velocidad, $\mathrm{m} / \mathrm{s}$ & $55 \pm 19$ & $65,8 \pm 18$ & $<0,001$ \\
Trabajo, $\mathrm{m}^{*} \mathrm{~kg}$ & $15932 \pm 5577$ & $25788 \pm 8371$ & $<0,001$ \\
DSP, $\mathrm{m} \%$ & $183,7 \pm 65$ & $326,7 \pm 94,6$ & $<0,001$ \\
\hline
\end{tabular}

fueron de $89 \pm 9 \%, 78 \pm 141 \mathrm{pm}, 17 \pm 2 \mathrm{rpm}, 121 \pm 16 \mathrm{~mm}$ $\mathrm{Hg}$ y $72 \pm 9 \mathrm{~mm} \mathrm{Hg}$, respectivamente (tabla 1 ).

Como se expresó antes, los sujetos se clasificaron como paradores $(n=30)$ y no paradores $(n=99)$ según la presencia o no de paradas. Las características basales para ambos grupos no demostraron diferencia estadísticamente significativa, a excepción del puntaje inicial de fatiga y la FR basal, que fueron mayores en el grupo que tuvo paradas (tabla 1).

Al finalizar la prueba, los puntajes de disnea y fatiga, así como las variables clínicas FC, FR y TAS final fueron significativamente mayores en el grupo que tuvo paradas, mientras que la $\mathrm{SaO}_{2}$ fue significativamente menor (tabla 1).

\section{Resultados nuevas variables derivadas de la PC6M}

Respecto a las determinaciones derivadas de la PC6M, los pacientes que pararon tuvieron distancia caminada, velocidad, trabajo, DDR y DSP significativamente menores que quienes no pararon, sin encontrar diferencia en cuanto al EID (tabla 2). La diferencia de la distancia caminada entre ambos grupos fue de $144 \pm 18$ metros, superior a los 26 metros que corresponden a la diferencia mínima clínicamente significativa de la prueba $(13,14)$.

\section{Resultados correlación y regresión de variables para paradas}

Se encontró correlación entre la presencia de paradas con los puntajes finales de disnea y fatiga, la distancia caminada, el trabajo de la caminata, el DDR y el DSP $(\mathrm{p}<0,001)$ (tabla 3$)$.
Tabla 3. Correlación de las paradas en la caminata de 6 minutos con variables demográficas y las medidas derivadas de la caminata de 6 minutos.

\begin{tabular}{|c|c|}
\hline Características & Paradas \\
\hline Edad, años & $-0,24$ \\
\hline Talla, cm & $-0,03$ \\
\hline Peso, kg & 0,05 \\
\hline $\mathrm{IMC}, \mathrm{kg} / \mathrm{m}^{2}$ & 0,05 \\
\hline Disnea inicial, Borg & $-0,35$ \\
\hline Disnea final, Borg & $-0,71$ \\
\hline Fatiga inicial, Borg & $-0,40$ \\
\hline Fatiga final, Borg & $-0,78$ \\
\hline $\mathrm{SaO}_{2}$ inicial, $\%$ & 0,18 \\
\hline $\mathrm{SaO}_{2}$ final, $\%$ & 0,49 \\
\hline FC inicial, Ipm & $-0,21$ \\
\hline FC final, Ipm & $-0,11$ \\
\hline FR inicial, rpm & $-0,42$ \\
\hline FR final, rpm & $-0,36$ \\
\hline TAS inicial, $\mathrm{mm} \mathrm{Hg}$ & 0,09 \\
\hline TAS final, $\mathrm{mm} \mathrm{Hg}$ & $-0,32$ \\
\hline TAD inicial, mm Hg & $-0,02$ \\
\hline TAD Final, mm Hg & $-0,15$ \\
\hline Distancia, $m \pm D E$ & 0,69 \\
\hline Velocidad, m/s & 0,33 \\
\hline Trabajo, m*kg & 0,65 \\
\hline DSP, $m \%$ & 0,78 \\
\hline EID & 0,14 \\
\hline
\end{tabular}


En el análisis de regresión, las variables que permanecieron significativas para la presencia de paradas en la caminata fueron saturación final (OR: 5,18; IC95\% 2,12- 12,6; $<<0,001$ ), distancia recorrida (OR: 11,4; IC95\% 4,23-30,8; $<<0,0001$ ), velocidad (OR: 3,80 (IC95\% 1,62-8.91; $<<0,002$ ), trabajo (OR: 9,14; IC95\% 3,52-23,70; $<<0,0001$ ) y DSP (OR 15,5; IC95\% 5,19-46,20; $<<0,001$ ).

En el modelo de regresión, las determinaciones con mayor significancia estadística fueron distancia, trabajo y DSP $(p<0,001)$ a las cuales se les cuantificó su capacidad operativa por medio del cálculo de área bajo la curva ROC (área bajo la curva de las características operativas del receptor), siendo de $0,83,0,83$ y 0,89 , respectivamente (figuras 1 a 3 ).

En el caso de la distancia, para un punto de corte $\leq 360$ metros, la sensibilidad fue de $88 \%$ (IC95\% 71-97) y la especificidad de 63\% (IC95\% 53-73) para predecir paradas (figura 1); mientras que para trabajo con un punto de corte de $\leq 22.620 \mathrm{~m} * \mathrm{~kg}$ la

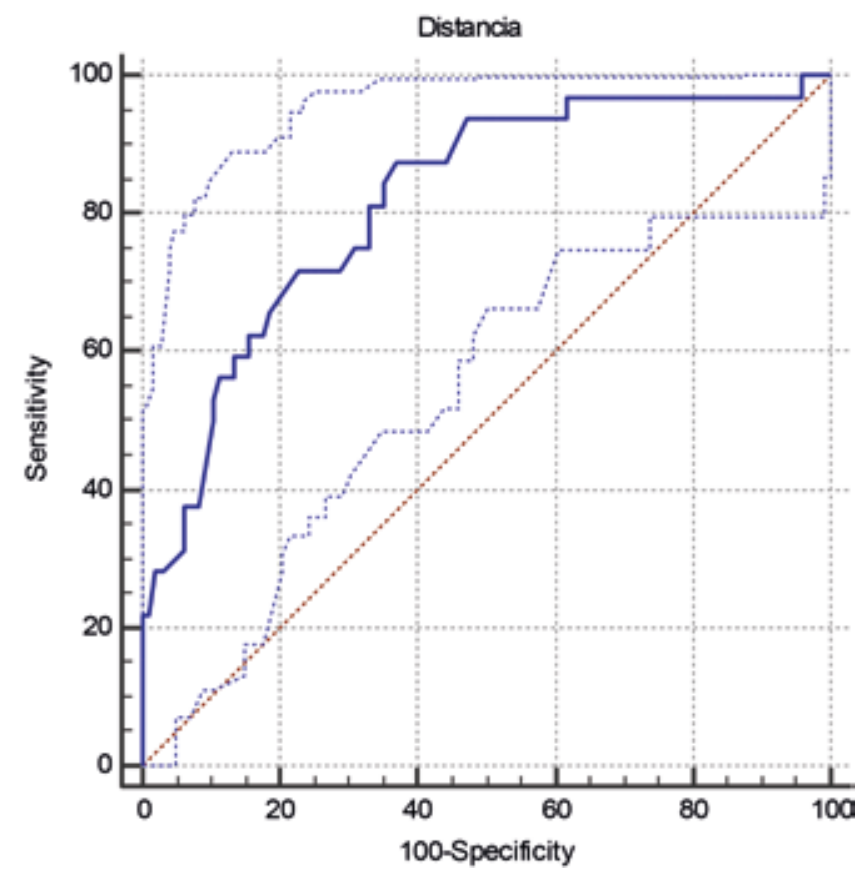

Figura 1. Curva ROC para distancia caminada como predictor de parada en caminata de 6 minutos en pacientes con EPOC. AUROC 0,81 (IC95\% 0,74-0,88; p < 0,0001; Punto de corte $\leq 360$ metros S: $88 \%$; E: $63 \%$ ). una sensibilidad fue de 91\% (IC95\% 75-98) y la especificidad de $62 \%$ (IC95\% 52-72) para predecir paradas (figura 2) y el DSP con un punto de corte $\leq 261 \mathrm{~m} \%$ con una sensibilidad de $88 \%$ (IC95\% 71-97) y especificidad de 75\% (IC95\% 66-84) para predecir paradas (figura 3).

\section{Discusión}

En el estudio se exploró la relación de variables demográficas y de mediciones de la PC6M incluyendo aquellas derivadas, recientemente propuestas, como velocidad, trabajo, DSP y EID con la presencia de paradas durante la PC6M en pacientes con EPOC. Al inicio de la prueba, el puntaje de fatiga y la FR fueron significativamente mayores en el grupo que hizo paradas, sin observar correlación posterior. Al finalizar la prueba, se encontró que los pacientes que tuvieron paradas durante la PC6M recorrieron menos distancia, con baja velocidad y menor trabajo, bajo DSP y menor $\mathrm{SO}_{2}$ al terminar, en comparación con quienes no pararon.

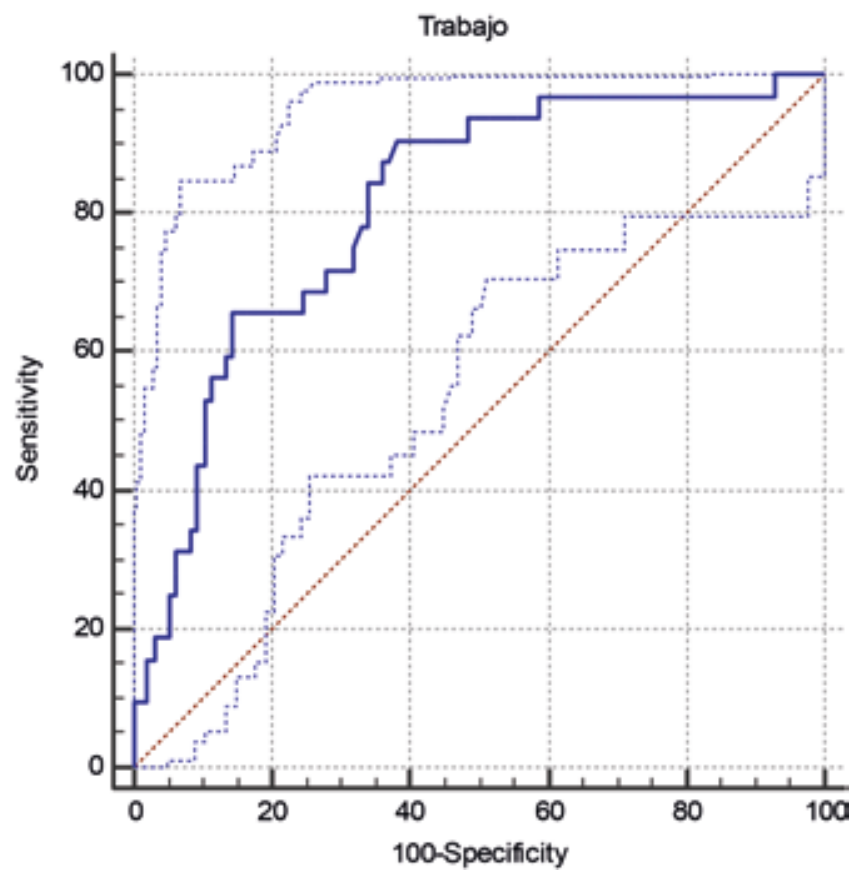

Figura 2. Curva ROC para trabajo como predictor de parada en caminata de 6 minutos en pacientes con EPOC. AUROC 0,81 (IC95\% 0,73-0,88; $p<0,0001$; Punto de corte $\leq 22.620$ kg*m S: 91\%; E: 62\%). 


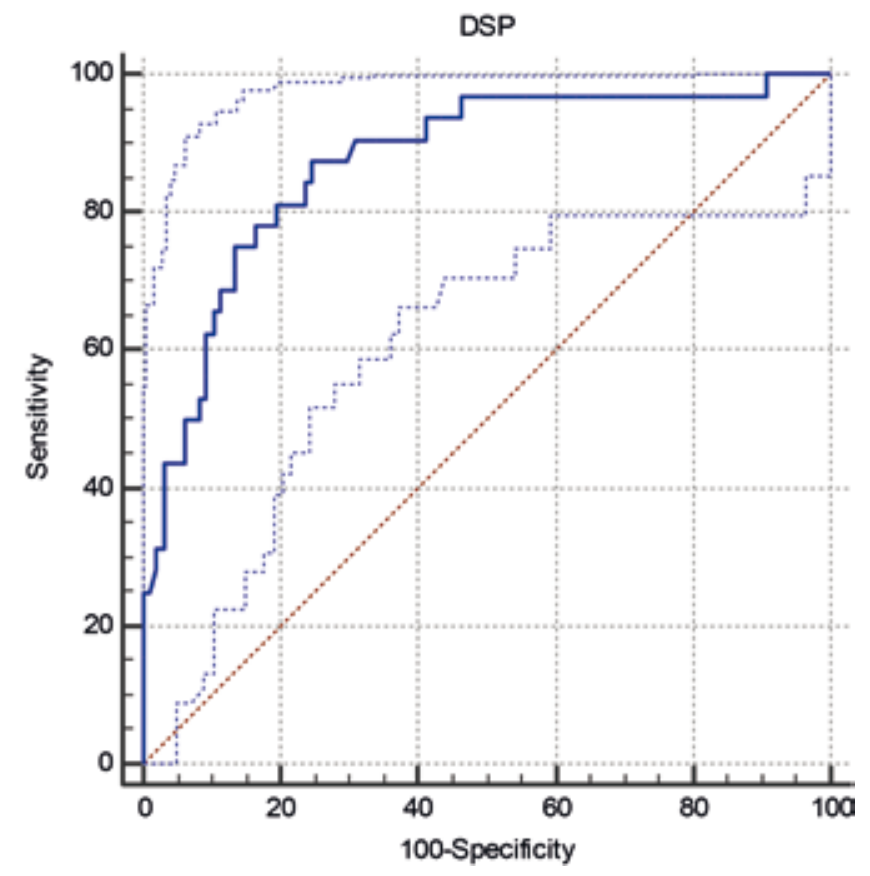

Figura 3. Curva ROC para DSP como predictor de parada en caminata de 6 minutos en pacientes con EPOC. AUROC 0,87 (IC95\% 0,80-0,92; p < 0,0001; Punto de corte $\leq 261$ $\mathrm{m} \% \mathrm{~S}: 88 \%$; E: $75 \%)$.

En este sentido, podrían considerarse las mediciones de DSP como una estimación directamente proporcional del metabolismo aeróbico durante la caminata y, por su parte, las mediciones de distancia, velocidad y trabajo, como una estimación directamente proporcional del gasto energético.

La PC6M es una prueba submáxima en la que se alcanza, hacia el tercer minuto, una meseta en el consumo de oxígeno, que se aproximaría al consumo pico logrado en una ergoespirometría con protocolo incremental, pero con una carga impuesta más baja en términos de producción de dióxido de carbono y respuesta ventilatoria. Para lograr esto el paciente adopta una velocidad constante que le permite completar el tiempo de caminata con una tasa de trabajo (distancia por peso corporal) baja, constante y sostenible sin llegar a la anaerobiosis (27).

El bajo valor de DSP es un reflejo del bajo trabajo (consumo de oxígeno) que se ve obligado a efectuar el paciente para cumplir el tiempo de 6 minutos a expensas de una menor distancia caminada. Las paradas durante la caminata pueden ser la consecuencia de anaerobiosis temprana dada la baja relación aporte de $\mathrm{O}_{2} /$ utilización de $\mathrm{O}_{2}$, de ahí la correlación encontrada de DSP con las paradas involuntarias durante la prueba.

En contraste, no se encontró correlación significativa con EID y la presencia de paradas durante la PC6M, posiblemente porque a la altura de Bogotá el punto de corte debería ser inferior a $88 \%$. Al tomarlo por debajo de $86 \%$, sí se observó correlación.

Andrianopoulos et al. han publicado el análisis más completo de la utilidad clínica de las nuevas variables derivadas de la PC6M en pacientes con EPOC, al analizar los datos de la cohorte ECLIPSE (17). Así mismo, el estudio reportó el valor pronóstico de las variables derivadas incluyendo distancia caminada, trabajo, velocidad, DSP y EID para la predicción de hospitalización y mortalidad, y señaló por primera vez que la presencia de paradas es un factor predictor independiente de riesgo de ambos desenlaces. No obstante, dicho trabajo no analizó las relaciones entre estas nuevas variables, fundamento necesario para determinar, en un estudio prospectivo, el valor pronóstico de la combinación de ellas (regla de predicción clínica).

Entre las fortalezas del estudio se destaca que se llevó a cabo un registro detallado de las paradas durante la PC6M, hecho que aún no se ha recomendado oficialmente en las guías vigentes (1214). En segundo lugar, se realizó un registro minuto a minuto de la $\mathrm{SaO}_{2}$, lo cual permitió realizar el cálculo de nuevos índices al finalizar la prueba. Así mismo, y tal como se recomienda en la última actualización de la PC6M $(13,14)$, en todos los pacientes se realizó una caminata de prueba, obviando las dificultades recientemente descritas por la variación en el cálculo de las nuevas determinaciones (17). Por último, la extracción de los datos fue hecha de forma independiente por dos de los autores.

No obstante, el estudio tiene algunas limitaciones. Primero, el número de sujetos reclutados, por lo que se requiere mayor población para confirmar los resultados. Y segundo, no existió información 
completa de variables de función pulmonar, exacerbaciones, puntajes multidimensionales o empleo de oxígeno, con el fin de ampliar los análisis de correlación y regresión.

A pesar de dichas limitaciones, este trabajo aporta información novedosa acerca de la correlación entre las paradas durante la PC6M y las nuevas variables derivadas del examen.

\section{Conclusión}

En el grupo evaluado de pacientes con EPOC, las variables derivadas de la PC6M como distancia, velocidad, trabajo y DSP, junto con la $\mathrm{SaO}_{2}$ al final de la prueba, muestran una correlación importante con la presencia de paradas en el examen, siendo distancia, trabajo y DSP las más importantes.

De tal forma, la medición de estas nuevas variables, así como el registro de paradas durante la PC6M, podría refinar la capacidad pronóstica de la prueba en pacientes con EPOC.

La incidencia de parada no deseada durante la PC6M podría indicar una etapa avanzada de gravedad o un mayor grado de actividad de la enfermedad, en la que la incorporación del paciente en un programa integral de rehabilitación pulmonar sería una estrategia deseable con el fin de reducir los riesgos de hospitalización y/o mortalidad.

\section{Agradecimientos}

Al Biólogo Carlos Fabián Morantes Ariza, MSc., de la Facultad de Biología de la Pontificia Universidad Javeriana, por su colaboración con el análisis estadístico de los datos, y a las enfermeras, terapeutas y a todo el personal de la unidad de neumología del Hospital Universitario San Ignacio.

\section{Nota}

Este artículo no genera conflicto de intereses. La información incluida en el manuscrito ha sido citada o se cuenta con permiso de los autores para su divulgación.

\section{Conflicto de intereses}

Este artículo no genera conflicto de intereses.

\section{Bibliografía}

1. Burney PG, Patel J, Newson R, Minelli C, Naghavi M. Global and regional trends in COPD mortality, 1990-2010. Eur Respir J. 2015;45:1239-47.

2. Caballero A, Torres-Duque CA, Jaramillo C, Bolívar F, Sanabria F, Osorio P, et al. Prevalence of COPD in five Colombian cities situated at low, medium, and high altitude (PREPOCOL study). Chest. 2008;133:343-9.

3. Londoño D, García M, Celis CA, Giraldo M et al. Guía de práctica clínica basada en la evidencia para la prevención, diagnóstico, tratamiento y seguimiento de la enfermedad pulmonar obstructiva crónica (EPOC) en población adulta. Acta Med Col. 2014;39(2-3S):5-48.

4. Celli BR, Cote CG, Marin JM, Casanova C, Montes de Oca M, Mendez RA, et al. The body-mass index, airflow obstruction, dyspnea, and exercise capacity index in chronic obstructive pulmonary disease. N Engl J Med. 2004;350:1005-12.

5. de Torres JP, Casanova C, Marín JM, Pinto-Plata V, Divo M, Zulueta JJ, et al. Prognostic evaluation of COPD patients: GOLD 2011 versus BODE and the COPD comorbidity index COTE. Thorax. 2014;69:799-804.

6. Faganello MM, Tanni SE, Sanchez FF, Pelegrino NR, Lucheta PA, Godoy I. BODE index and GOLD staging as predictors of 1-year exacerbation risk in chronic obstructive pulmonary disease. Am J Med Sci. 2010;339:10-4.

7. Marchand E. The BODE index as a tool to predict survival in COPD lung transplant candidates. Eur Respir J. 2010;36:1494-5.

8. Ramon MA, Gimeno-Santos E, Ferrer J, Balcells E, Rodríguez E, de Batlle J, et al; PAC-COPD Study Group. Hospital admissions and exercise capacity decline in patients with COPD. Eur Respir J. 2014;43:1018-27.

9. Trzaska-Sobczak M, Brożek G, Farnik M, Pierzchała W. Evaluation of COPD progression based on spirometry and exercise capacity. Pneumonol Alergol Pol. 2013;81:288-93.

10. Vogiatzis I, Zakynthinos S. Factors limiting exercise tolerance in chronic lung diseases. Compr Physiol. 2012;2:1779817.

11. Janaudis-Ferreira T, Beauchamp MK, Goldstein RS, Brooks D. How should we measure arm exercise capacity in patients with COPD? A systematic review. Chest. 2012;141:111-20.

12. ATS Committee on Proficiency Standards for Clinical Pulmonary Function Laboratories. ATS statement: guidelines for the six-minute walk test. Am J Respir Crit Care Med. 2002;166:111-7.

13. Singh SJ, Puhan MA, Andrianopoulos V, Hernandes NA, Mitchell KE, Hill CJ, et al. An official systematic review of the European Respiratory Society/American Thoracic Society: measurement properties of field walking tests in chronic respiratory disease. Eur Respir J. 2014;44:1447-78.

14. Holland AE, Spruit MA, Troosters T, Puhan MA, Pepin V, Saey D, et al. An official European Respiratory Society/Ame- 
rican Thoracic Society technical standard: field walking tests in chronic respiratory disease. Eur Respir J. 2014;44:142846.

15. Polkey MI, Spruit MA, Edwards LD, Watkins ML, Pinto-Plata V, Vestbo J, et al. Evaluation of COPD Longitudinally to Identify Predictive Surrogate Endpoints (ECLIPSE) Study Investigators. Six-minute-walk test in chronic obstructive pulmonary disease: minimal clinically important difference for death or hospitalization. Am J Respir Crit Care Med. 2013;187:382-6.

16. Chandra D, Wise RA, Kulkarni HS, Benzo RP, Criner G, Make B, et al; NETT Research Group. Optimizing the 6-min walk test as a measure of exercise capacity in COPD. Chest. 2012;142:1545-52.

17. Andrianopoulos V, Wouters EF, Pinto-Plata VM, Vanfleteren LE, Bakke PS, Franssen FM, et al. Prognostic value of variables derived from the six-minute walk test in patients with COPD: Results from the ECLIPSE study. Respir Med. 2015 Jun 25. [Epub ahead of print].

18. Studenski S, Perera S, Patel K, et al. Gait speed and survival in older adults. J. Am Med Assoc. 2011;30:50-8.

19. Ilgin D, Ozalevli S, Kilinc O, Sevinc C, Cimrin AH, Ucan ES. Gait speed as a functional capacity indicator in patients with chronic obstructive pulmonary disease. Ann Thorac Med. 2011;6:141-6.

20. Chuang ML, Lin IF, Wasserman K. The body weight-walking distance product as related to lung function, anaerobic threshold and peak VO2 in COPD patients. Respir Med. 2001;95:618-26.
21. Carter R, Holiday DB, Nwasuruba C, Stocks J, Grothues C, Tiep B. 6-minute walk work for assessment of functional capacity in patients with COPD. Chest. 2003;123:1408-15.

22. Alhamad EH, Shaik SA, Idrees MM, Alanezi MO, Isnani AC. Outcome measures of the 6 minute walk test: relationships with physiologic and computed tomography findings in patients with sarcoidosis. BMC Pulm Med. 2010;10:42.

23. Lettieri CJ, Nathan SD, Browning RF, Barnett SD, Ahmad S, Shorr AF. The distance-saturation product predicts mortality in idiopathic pulmonary fibrosis. Respir Med. 2006;100:173441.

24. Takigawa N, Tada A, Soda R, Date H, Yamashita M, Endo S, et al. Distance and oxygen desaturation in 6-min walk test predict prognosis in COPD patients. Respir Med. 2007;101:5617.

25. Casanova C, Cote C, Marin JM, Pinto-Plata V, de Torres JP, Aguirre-Jaime A, Vassaux C, Celli BR. and oxygen desaturation during the 6-min walk test as predictors of long-term mortality in patients with COPD. Chest. 2008;134:746-52.

26. Wong R, Sibley KM, Hudani M, Roeland S, Visconti M, Balsano J, Hill K, Brooks D. characteristics of people with chronic lung disease who rest during the six-minute walk test. Arch Phys Med Rehabil. 2010;91:1765-9.

27. Valero G, Bracciale P, Valerio F. Metabolic Requierements during six minutes walking tests in patients affected by chronic obstructive pulmonary disease in different stages. Open J Respir Dis. 2012;2:83-90. 